\title{
Guideline update for the performance of fusion procedures for degenerative disease of the lumbar spine. Part 5: Correlation between radiographic outcome and function
}

\author{
Sanjay S. Dhall, M.D., ${ }^{1}$ Tanvir F. Choudhri, M.D., ${ }^{2}$ Jason C. Eck, D.O., M.S., ${ }^{3}$ \\ Michael W. Groff, M.D., Zoher Ghogawala, M.D., ${ }^{5}$ William C. Watters III, M.D., ${ }^{6}$ \\ Andrew T. Dailey, M.D., ${ }^{7}$ Daniel K. Resnick, M.D., ${ }^{8}$ Alok Sharan, M.D., 9 \\ Praveen V. Mummaneni, M.D., Jeffrey C. Wang, M.D., ${ }^{10}$ and Michael G. Kaiser, M.D. ${ }^{11}$ \\ ${ }^{1}$ Department of Neurological Surgery, University of California, San Francisco, California; ${ }^{2}$ Department of \\ Neurosurgery, Icahn School of Medicine at Mount Sinai, New York, New York; ${ }^{3}$ Center for Sports Medicine \\ and Orthopaedics, Chattanooga, Tennessee; ${ }^{4}$ Department of Neurosurgery, Brigham and Women's Hospital, \\ Boston, Massachusetts; ${ }^{5}$ Alan and Jacqueline Stuart Spine Research Center, Department of Neurosurgery, \\ Lahey Clinic, Burlington, and Tufts University School of Medicine, Boston, Massachusetts; ${ }^{6}$ Bone and Joint \\ Clinic of Houston, Houston, Texas; ${ }^{7}$ Department of Neurosurgery, University of Utah, Salt Lake City, Utah; \\ ${ }^{8}$ Department of Neurosurgery, University of Wisconsin, Madison, Wisconsin; ${ }^{9}$ Department of Orthopaedic \\ Surgery, Montefiore Medical Center, Albert Einstein College of Medicine, Bronx, New York; ${ }^{10}$ Department of \\ Orthopaedic Surgery, Keck School of Medicine, University of Southern California, Los Angeles, California; \\ and ${ }^{11}$ Department of Neurosurgery, Columbia University, New York, New York
}

In an effort to diminish pain or progressive instability, due to either the pathological process or as a result of surgical decompression, one of the primary goals of a fusion procedure is to achieve a solid arthrodesis. Assuming that pain and disability result from lost mechanical integrity of the spine, the objective of a fusion across an unstable segment is to eliminate pathological motion and improve clinical outcome. However, conclusive evidence of this correlation, between successful fusion and clinical outcome, remains elusive, and thus the necessity of documenting successful arthrodesis through radiographic analysis remains debatable. Although a definitive cause and effect relationship has not been demonstrated, there is moderate evidence that demonstrates a positive association between radiographic presence of fusion and improved clinical outcome. Due to this growing body of literature, it is recommended that strategies intended to enhance the potential for radiographic fusion are considered when performing a lumbar arthrodesis for degenerative spine disease.

(http://thejns.org/doi/abs/10.3171/2014.4.SPINE14268)

KEY WORDS • fusion $•$ lumbar spine $\quad$ treatment outcomes
practice guidelines

\section{Recommendations}

\section{Grade B}

When performing lumbar arthrodesis for degenerative lumbar disease, strategies to achieve successful radiographic fusion should be considered, as there appears to be a correlation between successful fusion and improved clinical outcomes.

Abbreviations used in this paper: ALIF = anterior lumbar interbody fusion; DPQ = Dallas Pain Questionnaire; LBOS = Low Back Outcome Scale; LBPR = Low Back Pain Rating Scale; PLF = posterolateral lumbar fusion; VAS = visual analog scale.

\section{Rationale}

Achieving a solid arthrodesis following a spinal fusion procedure is generally believed to be an important goal; however, the relationship between successful fusion and clinical outcome has not been fully established. Therefore, the utility of exhaustive radiographic testing to determine fusion status may be questioned. The purpose of this review is to examine the literature regarding the relationship between fusion status and clinical outcome after lumbar arthrodesis procedures performed in the treatment of lumbar spinal degenerative disease. Additional information regarding the methodologies and criteria used to evaluate the evidence discussed below is 
located in the Methodology section of the first article in this issue (Part 1: Introduction and methodology). ${ }^{11}$

\section{Search Criteria}

For this update, a computerized search of the database of the National Library of Medicine between July 2003 and December 2011 was conducted using the search terms "lumbar spine fusion assessment," "lumbar spine pseudoarthrosis," or "lumbar spine fusion outcome." (The spelling "pseudoarthrosis" was used in searching, but searching on this spelling also retrieves publications with the spelling "pseudarthrosis.") The search was restricted to references in the English language involving humans. This yielded a total of 1076 references. The titles and abstracts of each of these references were reviewed. Papers not concerned with the assessment of postoperative fusion status or those not focused on adult degenerative lumbar disease (for example, papers focused on trauma-related fractures, infection, scoliosis, or isthmic spondylolisthesis) were discarded. Additional articles were obtained from the bibliographies of the selected articles. Fourteen new references were identified that provided either direct or supporting evidence relevant to the radiographic assessment of lumbar fusion status. These were considered in conjunction with the 37 references from the previous search from 1966 to July 2003. ${ }^{16}$ Reports involving Level III or better medical evidence are listed in Table 1. Supportive data are provided by additional references listed in the bibliography.

\section{Scientific Foundation}

Achievement of a solid fusion across the treated motion segments is an integral goal of any lumbar fusion procedure performed to treat low-back pain due to lumbar degenerative disease. Therefore, patients who achieve a solid fusion would be expected to have better clinical outcomes compared with those in whom osseous union does not occur (pseudarthrosis). However, a number of authors have described patients with pseudarthrosis with favorable clinical outcomes and patients with solid osseous unions who have poor clinical outcomes. ${ }^{3,7}$ The radiographic assessment of lumbar fusion status is imperfect and is not without potential downside to the patient (e.g., exposure to ionizing radiation) and society (e.g., health care resource utilization). If the clinical results associated with lumbar fusion procedures do not correlate with radiographic findings, one can question the utility of exhaustive radiographic study to demonstrate fusion. Furthermore, the incorporation of surgical techniques and adjuncts designed to increase radiographic fusion rates may be inappropriate unless a correlation between radiographic and clinical outcomes can be confirmed. The purpose of this document is to review the evidence for and against such a relationship.

A study correlating clinical outcomes with the results of the gold standard for assessment of lumbar fusion status (open surgical exploration) has not been performed. However, studies do exist in which investigators compared various radiographic fusion assessment techniques with clinical outcomes. In total, we noted 10 Level II and
III (4 Level II and 6 Level III) studies relating to correlation between clinical and radiographic outcome. Of these, 7 (3 Level II and 4 Level III) studies, showed a positive correlation between successful arthrodesis on radiographs and good clinical outcome. The remaining 3 studies did not show a positive correlation between radiographic fusion and good clinical outcome. We noted another 7 Level IV and V studies, and 5 of them did not show correlation between radiographic fusion and good clinical outcome. . $^{4,8-10,14,18,19}$

The Level II studies included the studies by Christensen et al. (2002), ${ }^{1}$ Kornblum et al. (2004), ${ }^{13}$ Kim et al. (2006), ${ }^{12}$ and Thalgott et al. (2009). ${ }^{17}$ In 2002, Christensen and colleagues published a prospective randomized 2-year follow-up study of 148 patients randomized to posterolateral lumbar fusion (PLF) plus pedicle screw fixation or anterior lumbar interbody fusion (ALIF), PLF, and pedicle screw fixation. ${ }^{1}$ Clinical outcome was assessed using the Dallas Pain Questionnaire (DPQ), the Low Back Pain Rating Scale (LBPR), and a work status survey. The authors found that patients in both treatment groups exhibited highly significant improvements in all 4 categories of quality of life (DPQ) as well as in the back pain and leg pain index (LBPR) compared with their preoperative status. They identified a significant positive relationship between fusion status and functional outcome: patients with successful radiographic fusion did significantly better than those without solid fusions on 3 of 4 subsections of the DPQ (there was also a nonsignificant improvement on the social concerns subsection).

Kornblum et al. ${ }^{13}$ retrospectively reviewed data from a randomized trial comparing instrumented to uninstrumented posterolateral lumbar fusion, and they looked specifically at the uninstrumented patients. They found that good/excellent outcomes in $86 \%$ of the patients with successful fusion versus $56 \%$ in those with pseudarthrosis (p $=0.01$ ), and similarly VAS scores (for both back pain and leg pain) were statistically higher in patients with successful fusion. It is unclear whether outcomes in patients with uninstrumented pseudarthrosis can be generalized to patients with instrumented pseudarthrosis. Kim et al., ${ }^{12}$ randomized a heterogeneous patient population to 1- or 2-level PLF, posterior lumbar interbody fusion (PLIF), or PLIF+PLF. They found that $91 \%$ of patients with fusion had superior clinical results as compared with $41 \%$ of patients with nonunion. Thalgott et al., ${ }^{17}$ randomized 50 patients undergoing ALIF with posterior instrumentation to receive either frozen or freeze-dried femoral allograft. In contrast to the previous 2 Level II studies, this study showed no statistically significant difference in ODI and VAS scores between patients with fusion and those with nonunion.

Of the 6 Level III studies, 4 showed a positive correlation between radiographic fusion and good clinical outcome: the 1995 study by Christensen et al. ${ }^{2}$ and the studies by Zdeblick, ${ }^{21}$ Wetzel et al., ${ }^{20}$ and Djurasovic et al. ${ }^{5}$ The remaining 2 studies - the study by Penta and Fraser ${ }^{15}$ and the study by Epstein ${ }^{6}$ - failed to show a correlation. Christensen et al. ${ }^{2}$ studied 120 consecutive patients who underwent ALIF. Clinical outcome was evaluated 5-13 years after surgery by using the DPQ. At 2 years postop- 
Part 5: Correlation between radiographic outcome and function

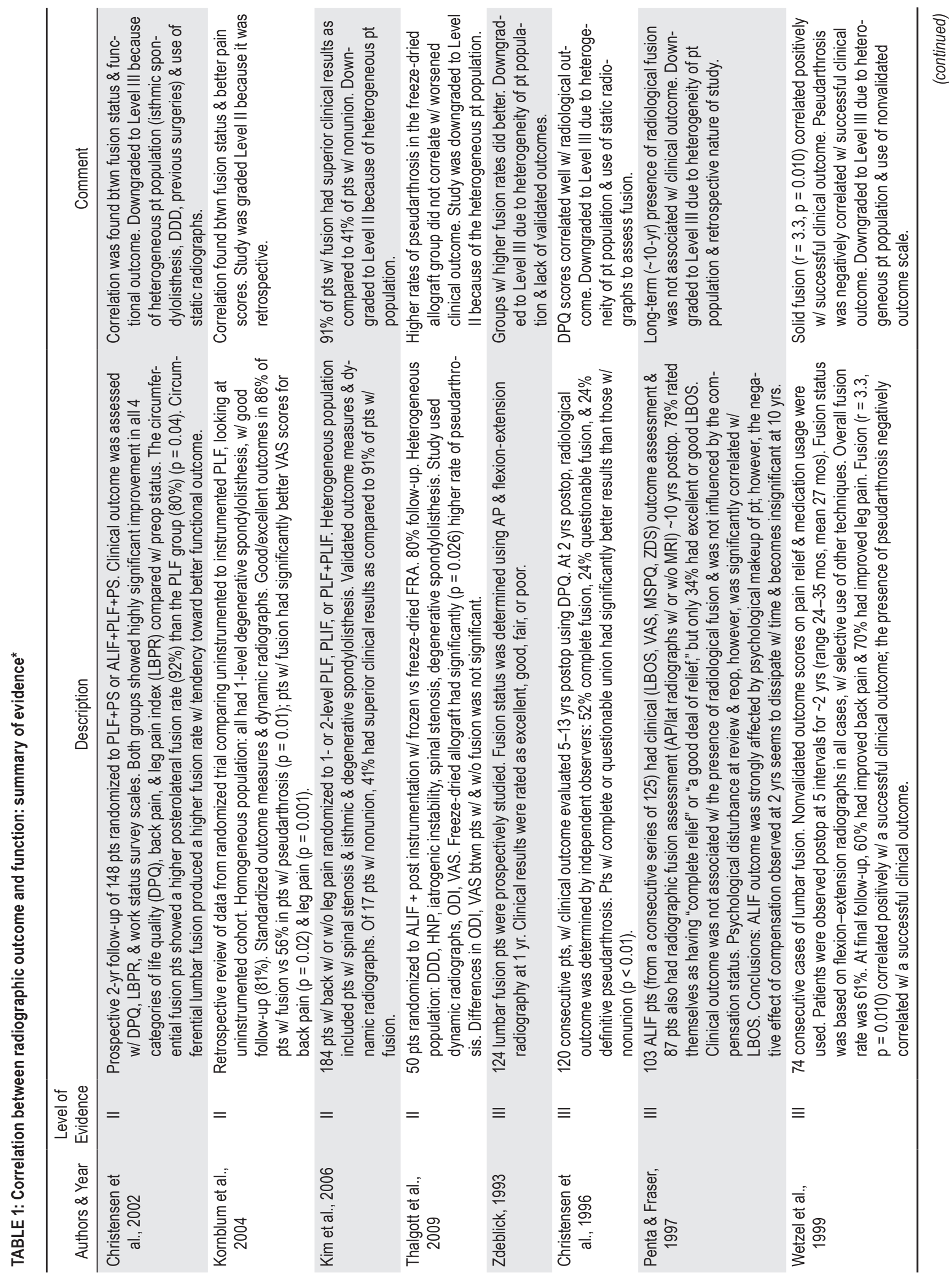




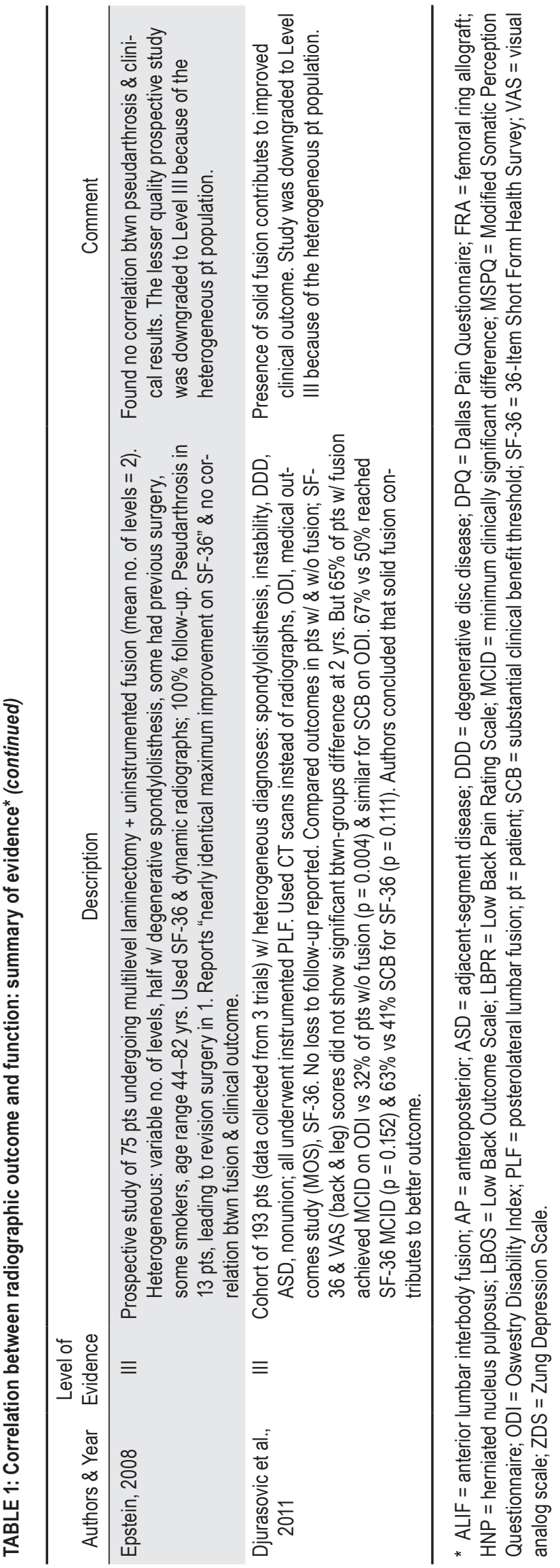

eratively, fusion outcome was assessed using static plain radiography assessed by independent observers. These authors reported complete fusion in $52 \%$ of patients, questionable fusion in 24\%, and definitive pseudarthrosis in $24 \%$. Patients with complete or questionable union had significantly better DPQ scores than those with nonunion $(\mathrm{p}<0.01)$. The authors concluded that DPQ scores correlated well with radiological outcome. This study is considered to provide Level III medical evidence supporting fusion status as a predictor of functional outcome because the radiographic and clinical follow-up evaluations were obtained at widely separated time points (between 3 and 11 years apart) and because the study relied on static plain radiography to determine fusion status and this modality has been shown to have limited accuracy. ${ }^{16}$

Wetzel and colleagues prospectively evaluated 74 consecutive patients who underwent lumbar fusion. ${ }^{20}$ Outcomes were measured using subjective clinical outcome scores pertaining to pain relief and medication usage. The patients were observed at 5 intervals after surgery during a minimum 2-year follow-up period (range 24-35 months, mean 27 months). Fusion status was evaluated using lateral flexion-extension radiography in all cases, with the selective use of other techniques. The authors noted a $61 \%$ fusion rate. At final follow-up examination, $60 \%$ of patients had improvement in back pain and 70\% had improvement in leg pain. The presence of radiographic fusion correlated positively with a successful clinical outcome $(r=3.3, p=0.010)$. Similarly, in a prospective study of 124 lumbar fusion patients assigned to 3 different surgical treatment groups, Zdeblick assessed fusion status by performing static and flexion-extension lateral radiography at 1 year. ${ }^{21}$ The clinical outcomes were rated as excellent, good, fair, or poor. The study showed that patients in the groups with higher fusion rates had better clinical outcomes. These studies, although prospective, are considered to provide Level III medical evidence in support of the correlation between radiographic and clinical outcome because of the use of nonvalidated clinical outcome measures. ${ }^{19}$

Djurasovic et al. ${ }^{5}$ studied data on 193 patients collected from 3 clinical trials in which the patients underwent instrumented PLF for diverse indications. The authors compared outcomes in the patients with fusion versus those with nonunion and found that $65 \%$ of the patients with fusion achieved MCID on the ODI as compared with $32 \%$ of those with nonunion (a statistically significant difference).

In contrast, other studies have failed to demonstrate a statistically significant correlation between clinical and radiographic outcome in patients following lumbar arthrodesis surgery.

In a long-term outcome study ( $>10$ years), Penta and Fraser $^{15}$ reported on 103 patients who underwent ALIF (from a consecutive series of 125 cases). Clinical outcome assessment involved various validated outcome measures, including the Low Back Outcome Scale (LBOS). Eighty-seven patients also underwent fusion assessment with anteroposterior and lateral radiography. The authors reported that $78 \%$ of patients rated themselves as having “complete relief” or "a good deal of relief," but only $34 \%$ 


\section{Part 5: Correlation between radiographic outcome and function}

had excellent or good LBOS scores. The patients' clinical outcomes could not be correlated with the presence of radiographic fusion. This study also provides Level III medical evidence against a correlation between radiographic fusion status and clinical outcome following lumbar fusion surgery.

Similarly, Epstein ${ }^{6}$ prospectively studied 75 patients with heterogeneous diagnoses who underwent multilevel decompression and uninstrumented fusion. She reported "nearly identical maximum improvement of SF-36" and that there was not a correlation between radiographic fusion and good clinical outcome.

\section{Summary}

There are a total of 10 Level II and III studies regarding this topic. Of these, 7 showed a positive correlation between radiographic presence of fusion and good clinical outcome. Based on the North American Spine Society (NASS) criteria used in the methodology for these guidelines, these are sufficient data to make a Grade B recommendation that strategies that lead to successful radiographic fusion lead to improved clinical outcomes.

\section{Key Issues for Further Investigation}

A prospective observational study involving categorization of patients based on multiple validated outcome instrument-derived outcomes and multimodal radiographic outcome assessment would provide Level II medical evidence supporting or refuting the importance of radiographic fusion.

\section{Acknowledgments}

We would like to acknowledge the AANS/CNS Joint Guidelines Committee (JGC) for their review, comments, and suggestions, Laura Mitchell, CNS Guidelines Project Manager, for her organizational assistance and Linda O'Dwyer, medical librarian, for assistance with the literature searches. We would also like to acknowledge the following individual JGC members for their contributions throughout the review process: Timothy Ryken, M.D.; Kevin Cockroft, M.D.; Sepideh Amin-Hanjani, M.D.; Steven N. Kalkanis, M.D.; John O'Toole, M.D., M.S.; Steven Casha, M.D. Ph.D.; Aaron Filler, M.D., Ph.D., F.R.C.S.; Daniel Hoh, M.D.; Steven Hwang, M.D.; Todd McCall, M.D.; Jeffrey J. Olson, M.D.; Julie Pilitsis, M.D., Ph.D.; Joshua Rosenow, M.D.; and Christopher Winfree, M.D.

\section{Disclosure}

Administrative costs of this project were funded by the Congress of Neurological Surgeons and the Joint Section on Disorders of the Spine and Peripheral Nerves of the American Association of Neurological Surgeons and Congress of Neurological Surgeons. No author received payment or honorarium for time devoted to this project. Dr. Ghogawala receives grants from the Patient Centered Outcomes Research Institute (PCORI) and the National Institutes of Health (NIH). Dr. Groff is a consultant for DePuy Spine and EBI Spine. Dr. Mummaneni owns stock in Spinicity and receives honoraria from DePuy Spine and Globus and royalties from DePuy Spine, Quality Medical Publishers, and Thieme Publishing. Dr. Wang owns stock in Bone Biologics, AxioMed, Amedica, CoreSpine, Expanding Orthopedics, Pioneer, Syndicom, VG Innovations, PearlDiver,
Flexuspine, Axis, FzioMed, Benvenue, Promethean, Nexgen, ElectroCore, and Surgitech and holds patents with and receives royalties from Biomet, Stryker, SeaSpine, Aesculap, Osprey, Amedica, Synthes, and Alphatec. The authors report no other potential conflicts of interest concerning the materials or methods used in this study or the findings specified in this paper.

Author contributions to the study and manuscript preparation include the following. Acquisition of data: all authors. Analysis and interpretation of data: all authors. Drafting the article: Dhall. Critically revising the article: all authors. Reviewed submitted version of manuscript: all authors. Approved the final version of the manuscript on behalf of all authors: Dhall. Study supervision: Kaiser.

\section{References}

1. Christensen FB, Hansen ES, Eiskjaer SP, Høy K, Helmig P, Neumann P, et al: Circumferential lumbar spinal fusion with Brantigan cage versus posterolateral fusion with titanium Cotrel-Dubosset instrumentation: a prospective, randomized clinical study of 146 patients. Spine (Phila Pa 1976) 27:2674-2683, 2002

2. Christensen FB, Karlsmose B, Hansen ES, Bünger CE: Radiological and functional outcome after lumbar interbody spinal fusion. Eur Spine J 5:293-298, 1996

3. DePalma AF, Rothman RH: The nature of pseudoarthrosis. 1968. Clin Orthop Relat Res (284):3-9, 1992

4. Diedrich O, Perlick L, Schmitt O, Kraft CN: Radiographic characteristics on conventional radiographs after posterior lumbar interbody fusion: comparative study between radiotranslucent and radiopaque cages. J Spinal Disord 14:522532, 2001

5. Djurasovic M, Glassman SD, Dimar JR II, Howard JM, Bratcher KR, Carreon LY: Does fusion status correlate with patient outcomes in lumbar spinal fusion? Spine (Phila Pa 1976) 36: 404-409, 2011

6. Epstein NE: An analysis of noninstrumented posterolateral lumbar fusions performed in predominantly geriatric patients using lamina autograft and beta tricalcium phosphate. Spine J 8:882-887, 2008

7. Flatley TJ, Derderian H: Closed loop instrumentation of the lumbar spine. Clin Orthop Relat Res (196):273-278, 1985

8. Gaetani P, Aimar E, Panella L, Levi D, Tancioni F, Di Ieva A, et al: Functional disability after instrumented stabilization in lumbar degenerative spondylolisthesis: a follow-up study. Funct Neurol 21:31-37, 2006

9. Greenough CG, Peterson MD, Hadlow S, Fraser RD: Instrumented posterolateral lumbar fusion. Results and comparison with anterior interbody fusion. Spine (Phila Pa 1976) 23:479_ 486, 1998

10. Hackenberg L, Halm H, Bullmann V, Vieth V, Schneider M, Liljenqvist U: Transforaminal lumbar interbody fusion: a safe technique with satisfactory three to five year results. Eur Spine J 14:551-558, 2005

11. Kaiser MG, Eck JC, Groff MW, Watters WC III, Dailey AT, Resnick DK, et al: Guideline update for the performance of fusion procedures for degenerative disease of the lumbar spine. Part 1: Introduction and methodology. J Neurosurg Spine 21: 2-6, 2014

12. Kim KT, Lee SH, Lee YH, Bae SC, Suk KS: Clinical outcomes of 3 fusion methods through the posterior approach in the lumbar spine. Spine (Phila Pa 1976) 31:1351-1358, 2006

13. Kornblum MB, Fischgrund JS, Herkowitz HN, Abraham DA, Berkower DL, Ditkoff JS: Degenerative lumbar spondylolisthesis with spinal stenosis: a prospective long-term study comparing fusion and pseudarthrosis. Spine (Phila Pa 1976) 29:726-734, 2004

14. Lee CS, Hwang CJ, Lee SW, Ahn YJ, Kim YT, Lee DH, et al: Risk factors for adjacent segment disease after lumbar fusion. Eur Spine J 18:1637-1643, 2009 
15. Penta M, Fraser RD: Anterior lumbar interbody fusion. A minimum 10-year follow-up. Spine (Phila Pa 1976) 22:24292434, 1997

16. Resnick DK, Choudhri TF, Dailey AT, Groff MW, Khoo L, Matz PG, et al: Guidelines for the performance of fusion procedures for degenerative disease of the lumbar spine. Part 5: correlation between radiographic and functional outcome. J Neurosurg Spine 2:658-661, 2005

17. Thalgott JS, Fogarty ME, Giuffre JM, Christenson SD, Epstein AK, Aprill C: A prospective, randomized, blinded, single-site study to evaluate the clinical and radiographic differences between frozen and freeze-dried allograft when used as part of a circumferential anterior lumbar interbody fusion procedure. Spine (Phila Pa 1976) 34:1251-1256, 2009

18. Tokuhashi Y, Matsuzaki H, Oda H, Uei H: Clinical course and significance of the clear zone around the pedicle screws in the lumbar degenerative disease. Spine (Phila Pa 1976) 33:903908,2008

19. Vamvanij V, Fredrickson BE, Thorpe JM, Stadnick ME, Yuan
HA: Surgical treatment of internal disc disruption: an outcome study of four fusion techniques. J Spinal Disord 11:375-382, 1998

20. Wetzel FT, Brustein M, Phillips FM, Trott S: Hardware failure in an unconstrained lumbar pedicle screw system. A 2-year follow-up study. Spine (Phila Pa 1976) 24:1138-1143, 1999

21. Zdeblick TA: A prospective, randomized study of lumbar fusion. Preliminary results. Spine (Phila Pa 1976) 18:983-991, 1993

Manuscript submitted March 13, 2014.

Accepted April 1, 2014.

Please include this information when citing this paper: DOI: 10.3171/2014.4.SPINE14268.

Address correspondence to: Michael G. Kaiser, M.D., Columbia University, Neurological Surgery, The Neurological Institute, 710 W. 168th St., New York, NY 10032. email: mgk7@columbia.edu. 